\title{
Systemwechsel in der Zusatzversorgung - Ursachen, Umsetzung und Auswirkung auf die Finanzierung
}

Die Zusatzversorgung im öffentlichen Dienst ist das größte System der betrieblichen Alterssicherung in Deutschland. Zur Jahrtausendwende wurde die nachhaltige Finanzierbarkeit nach dem damaligen Leistungsrecht immer fraglicher, sodass sich die Tarifparteien auf eine grundlegende Reform der zusätzlichen Alterssicherung im öffentlichen Dienst einigten. Sie erweist sich als pragmatische und transparente Lösung mit Blick auf die zukünftige finanzielle Entwicklung der Zusatzversorgungskassen, belastet jedoch vornehmlich die Arbeitnehmerseite. ${ }^{1}$

\section{Einleitung}

Die tarifvertraglich Beschäftigen des öffentlichen Dienstes bekommen neben ihrer regulären Entlohnung eine zusätzliche Alterssicherung gewährt. Durch diese erhalten die verrenteten Arbeitnehmer zur gesetzlichen Rente eine Betriebsrente, die ihnen hilft, ihren Lebensstandard im Alter zu sichern. Neben der hohen Arbeitsplatzsicherheit ist die zusätzliche Alterssicherung ein wichtiges Argument für die Personalgewinnung im öffentlichen Dienst (Seiter 2003, S. 188). Zudem wird die Bedeutung von Betriebsrenten in der Wahrnehmung der Arbeitnehmer durch die Niveauabsenkung im Bereich der gesetzlichen Rente im Zuge der Riesterreform weiter steigen. Mit der Zusatzversorgung gewinnt der Staat als Arbeitgeber nicht nur an Attraktivität, sondern er gilt der Privatwirtschaft auch als Vorbild für die Einführung von Betriebsrenten.

Bis zum Jahr 2001 wurde den tarifvertraglich Beschäftigen eine zusätzliche Alterssicherung im Rahmen der Gesamtversorgung gewährt, jedoch drohten die daraus resultierenden Kosten, die finanzielle Stabilität der Zusatzversorgung zu gefährden. Unter erheblichem Zeitdruck einigten sich die Tarifparteien damals auf eine tief greifende Reform der Zusatzversorgung, die in die Ablösung der Gesamtversorgung durch ein sogenanntes Versorgungspunktemodell mündete. Der vorliegende Beitrag beschreibt zunächst diesen Wandel und erklärt dessen Ursachen (Abschnitte 2-5). Daran anschließend wird der Einfluss des Systemwechsels auf die zukünftige finan- zielle Entwicklung der Zusatzversorgung im öffentlichen Dienst analysiert (Abschnitt 6). Ein knappes Fazit fasst die Ergebnisse zusammen und wirft die Frage nach der Nachhaltigkeit des neuen Systems auf (Abschnitt 7).

\section{2 \\ Das System der Gesamt- versorgung bis 2001}

Im Jahr 1967 wurde im Bereich der Zusatzversorgung des öffentlichen Dienstes das Gesamtversorgungssystem eingeführt. Den Arbeitnehmern sollte dadurch eine Altersvorsorge, die sich an den Pensionen der Beamten orientiert, gewährt werden. Die Leistungszusage der Zusatzversorgung zielte demnach, genau wie im Bereich der Beamtenversorgung, auf die Sicherung eines gewissen Versorgungsniveaus ab. Das zugesagte Versorgungsniveau ergab sich dabei als bestimmter Prozentsatz der letzten Bezüge der Versicherten vor Renteneintritt. Welcher Anteil des Endgehalts den Versicherten im Rahmen der Gesamtversorgung zugesagt wurde, hing im Wesentlichen von der Zeit ab, die diese im öffentlichen Dienst beschäftigt waren.

Im Gegensatz zu ihren verbeamteten Kollegen sind die tarifvertraglich Beschäftigten des öffentlichen Dienstes in der gesetzlichen Rentenversicherung pflichtversichert. Die gesetzliche Rente deckt dadurch bereits einen Teil des zugesagten Versorgungsniveaus ab, sodass die Zusatzversorgung zu Zeiten der Gesamtversorgung die Lücke zwischen gesetzlicher Rente und zugesprochenem Versorgungsniveau zu füllen hatte (Fieberg 2002, S. 231). Die von den Zusatzversorgungskassen ausgezahlten Versorgungsrenten ergaben sich demnach als Differenz zwischen dem individuell erreichten Gesamtversorgungsniveau und der darauf anzurechnenden gesetzlichen Rente.

In einem ersten Schritt wurde das individuell erreichte Gesamtversorgungsniveau des Versicherten nach folgender Formel ermittelt:

Gesamtversorgung $=($ fiktives Netto- $)$ Endeinkommen $\times$ versorgungsfähige Zeit $\times$ jährlicher Versorgungssatz

$\mathrm{Zu}$ seiner Berechnung legten die Kassen zunächst das durchschnittliche BruttoEntgelt der letzten drei Jahre vor Renteneintritt zugrunde, da sich die Leistungszusage auf das Endgehalt bezog. Zur Vermeidung einer Überversorgung zog die Kasse von diesem durchschnittlichen Brutto-Entgelt in einem pauschalierenden Verfahren Steuern und Sozialabgaben ab, wodurch sich ein fiktives, durchschnittliches Netto-Entgelt ergab (Beckmann/Hebler 2003, S. 67). Anhand des Versorgungssatzes wurde festgelegt, welcher Anteil des Endge-

\footnotetext{
Der vorliegende Beitrag folgt einem Call for Papers der WSI-Mitteilungen zum Thema "Der Staat als Arbeitgeber", der unter www.boeckler.de/pdf/ wsimit_2009_call_for_paper_ag_staat.pdf abgerufen werden kann.
}

Stefan Preller, Dipl. Volkswirt, ist Forschungsreferent am Deutschen Forschungsinstitut für öffentliche Verwaltung in Speyer. Arbeitsschwerpunkte: Föderalismus, Staatsverschuldung, Altersvorsorge. e-mail: Preller@foev-speyer.de 
halts als Gesamtversorgung gewährt wurde. Er bestimmte sich aus der versorgungsfähigen Zeit, die einerseits Zeiten enthielt, die der Versicherte im öffentlichen Dienst beschäftigt war (Umlagemonate), und andererseits wurden auch hälftig die Zeiten der gesetzlichen Rentenversicherung berücksichtigt, die nicht zugleich Umlagemonate waren (sogenannte Halbanrechnung der Vordienstzeiten). Nach 40 Jahren konnte ein maximaler Versorgungssatz von $91,25 \%$ des fiktiven Netto-Endeinkommens erreicht werden (bzw. $75 \%$ des Brutto-Endeinkommens, falls dieser Wert niedriger war). Pro Jahr ergab sich durch die lineare Staffelung ein Versorgungssatz von $2,294 \%(1,875 \%$ bei der BruttoGesamtversorgung). Nach einer fünfjährigen Wartezeit wurde eine Mindestversorgung in Höhe von 45 \% des fiktiven NettoEndeinkommens bzw. 35 \% des BruttoEndeinkommens zugebilligt.

Der zweite Schritt zur Ermittlung der Zusatzrente bestand in der Anrechnung der gesetzlichen Rente auf die Gesamtversorgung. Diese Anrechnung erfolgte nach Einführung der sogenannte Spitzanrechnung fortlaufend auch während des Rentenbezugs (Kiefer 1996, S. 28). Damit wirkten sich Änderungen in der Höhe der gesetzlichen Rente unmittelbar auf die Höhe der Zusatzrenten aus. Bei sinkenden gesetzlichen Renten mussten die Versorgungsrenten steigen, um das zugesagte Versorgungsniveau beizubehalten.

Die Dynamisierung der Versorgungsrenten folgte der Anpassung der Beamtenpensionen. Somit wurden die Versorgungsrenten nicht direkt dynamisiert, sondern es wurde die zugrunde liegende Gesamtversorgung entsprechend angepasst. Bei diesen Anpassungen wurden auch Änderungen im Steuer- und Sozialabgabenrecht, die sich über die Nettobegrenzung des betrachteten Endgehalts auf die Gesamtversorgung auswirkten, berücksichtigt. Somit wirkten sich Gesetzesänderungen im Bereich der Steuern und Sozialabgaben auch auf die Höhe der laufenden Versorgungsrenten aus.

Eine an der Gesamtversorgung orientierte Versorgungsrente gewährten die Kassen nur, wenn der Arbeitnehmer bis zum Eintritt des Versicherungsfalles im öffentlichen Dienst beschäftigt und dadurch pflichtversichert war. Arbeitnehmer, die vorzeitig aus dem öffentlichen Dienst ausschieden, verloren diesen Anspruch. Stattdessen erhielten sie eine sogenannte Versi- cherungsrente, ${ }^{2}$ die im Normalfall wesentlich geringer war und nicht dynamisiert wurde. Durch diese Regelung sollte der Verlust der Versorgungsansprüche bei einer vorzeitigen Beendigung des Beamtenverhältnisses nachgezeichnet werden (Beckmann/Hebler 2008, S. 72).

\section{3 \\ Notwendigkeit des Systemwechsels}

Die Gründe, die zur Abkehr vom System der Gesamtversorgung in der Zusatzversorgung des öffentlichen Dienstes führten, sind vielschichtig. Hier spielten mehrere Entwicklungen zusammen, die in ihrer Summe zu einem starken Anstieg des Umlagesatzes geführt hätten. Diese Entwicklungen betrafen drei Bereiche:

\subsection{ABHÄNGIGKEITEN ZU ÄUßEREN BEZUGSSYSTEMEN}

In der Gesamtversorgung bestanden systembedingt zahlreiche Abhängigkeiten zu äußeren Bezugssystemen wie der Beamtenversorgung, dem Steuer- und Sozialabgabenrecht sowie zur gesetzlichen Rente. Änderungen in diesen Systemen beeinflussten die Höhe der Zusatzrenten, ohne dass die Tarifparteien sie kontrollierten. So senkte die Rentenanpassungsverordnung 2000 schrittweise das Niveau der gesetzlichen Rente. Bei gleichbleibender Gesamtversorgungszusage hätten die Versorgungsrenten angehoben werden müssen, um das zugebilligte Versorgungsniveau beibehalten zu können. Neben dem kostensteigernden Effekt blieb die Frage, wie es $\mathrm{zu}$ rechtfertigen sei, dass dadurch die Beschäftigten des öffentlichen Dienstes von der Niveauabsenkung in der gesetzlichen Rente unberührt blieben. Ein ähnlicher Effekt hinsichtlich der Kostenentwicklung ging von den Steuerentlastungsgesetzen der Ära Schröder aus, da sie die NettoGesamtversorgung erhöhten, wodurch wiederum die auszuzahlenden Versorgungsrenten stiegen. Ferner diente die Einführung der Ökosteuer im Jahr 1999 teilweise der Reduzierung des Rentenversicherungsbeitrags, auch hierdurch erhöhte sich das fiktive Netto-Endgehalt, das der Berechnung der Versorgungsrenten zugrunde lag (Langenbrinck/Mühlstedt 2007, S. 3).

\subsection{DEMOGRAFIE UND VERSICHERTENSTRUKTUR}

Wie andere Rentensysteme auch, wird die Zusatzversorgung durch den demografischen Wandel belastet. Die steigende Lebenserwartung der Bevölkerung führt zu längeren Rentenbezugszeiten, wodurch die Anzahl der Renten im System steigt (Ehrentraut/Raffelhüschen 2008, S. 518), auch in Relation zu den aktiv Beschäftigen. Im Falle der Zusatzversorgung erhöht sich der Rentnerquotient jedoch nicht nur aufgrund der Demografie. Die unstetige Personalpolitik im öffentlichen Dienst der Vergangenheit wird in den nächsten Dekaden ebenfalls zu einem steigenden Rentneranteil in der Zusatzversorgung führen. Diese Entwicklung ist der starken Personalexpansion seit den 1970er Jahren und der späteren Personalreduktion seit Beginn der 1990er Jahre geschuldet (Heubeck/ Rürup 2000, S. 60). Außerdem wurde diese Entwicklung durch das sinkende durchschnittliche Renteneintrittsalter in der $\mathrm{Zu}$ satzversorgung begünstigt (Deutscher Bundestag 2005, S. 248). Die Auseinanderentwicklung von Rentenzahl und aktiv Beschäftigten wirkt sich besonders in umlagefinanzierten Alterssicherungssystemen, so auch in der Zusatzversorgung, negativ aus. Abbildung 1 veranschaulicht die scherenartige Entwicklung von Aktiven und Rentnern. Insofern sich die Löhne und die Rentenhöhen zumindest ähnlich entwickeln, führt die dargestellte Situation zu steigenden Umlagesätzen, wodurch die Lohnkosten im öffentlichen Dienst ebenfalls steigen.

\subsection{RECHTSPRECHUNG}

Ein weiterer Bereich, der entscheidend zur Ablösung des Gesamtversorgungssystems beitrug, war die höchstrichterliche Rechtsprechung zur Zusatzversorgung im öffentlichen Dienst. Im Vorfeld des Systemwechsels ergingen mehrere Urteile, deren Umsetzungen mit teilweise erheblichen Kostensteigerungen verbunden gewesen wären und die von den Tarifparteien so nicht erwartet werden konnten (Kiefer 1996, S. 39). Neben den Urteilen zur fehlenden Dynamisierung der Mindestver-

\footnotetext{
2 Die Versicherungsrente errechnete sich aus 1,25\% der Pflichtbeiträge, die vor dem 01.01.1978 entrichtet wurden, sowie 0,03125\% der Entgelte, für die in der Folgezeit Umlagen entrichtet wurden.
} 


\section{Abb. 1: Entwicklung der Pflichtversicherten und Renten aus}

Pflichtversicherung der VBL ${ }^{1}$ zum Basisjahr 1990 - in \% -

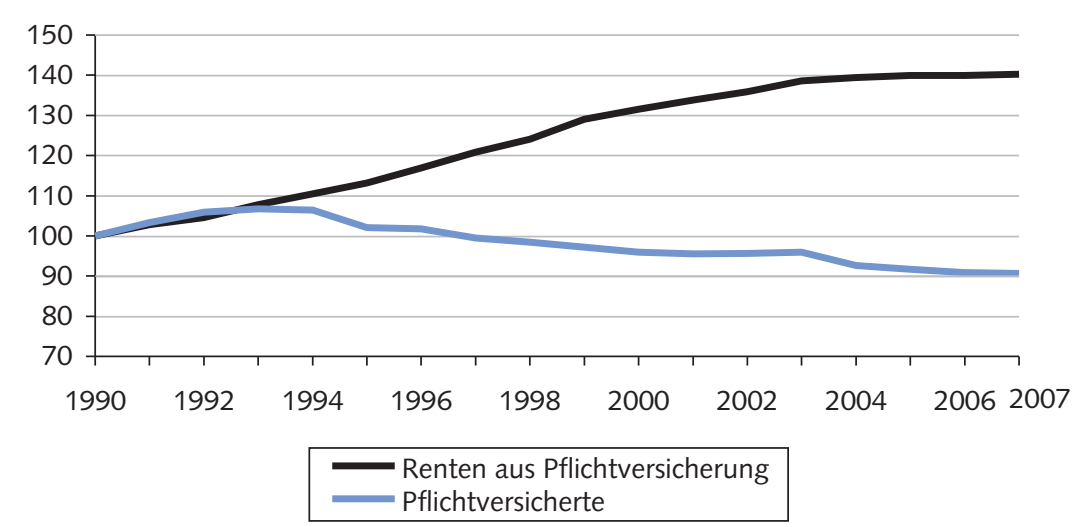

'Versorgungskasse des Bundes und der Länder (Abrechnungsverband West).

sorgungsrenten (BVerfG, 1 BvR 1554/89 vom 15.7.1998) und zur Zusatzversorgung der Teilzeitbeschäftigten (BVerfG, $1 \mathrm{BvR}$ 1246/95 vom 25.8.1999) erwies sich vor allem die mögliche Umsetzung des Urteils zur Halbanrechung der Vordienstzeiten bei gleichzeitiger Vollanrechnung der darauf zurückzuführenden gesetzlichen Renten (BVerfG, 1 BvR 1136/96 vom 22.3.2000) als besonders kostenintensiv (Stephan 2002, S. 50). Bei letzterem Urteil hielt das Bundesverfassungsgericht außerdem fest, dass die Rechtsmaterie der Zusatzversorgung hinsichtlich ihrer Komplexität bereits an verfassungsmäßige Grenzen stoße. Weitere Regelungen hätten die Materie nicht verkomplizieren dürfen.

Zusammengenommen führten die Entwicklungen in der gesetzlichen Rente, im Steuer- und Sozialabgabenrecht hinsichtlich der Demografie und der Versichertenstruktur sowie der Rechtsprechung zu einem erheblichen Druck auf die Umlagesätze der Zusatzversorgungskassen. Bei der größten Kasse, der Versorgungsanstalt des Bundes und der Länder (VBL), musste im ungünstigsten Fall mit einem Umlagesatz von $15,4 \%$ gerechnet werden (Stephan 2002, S. 49). Im Vergleich zum Jahr 1998 wäre dies ein Anstieg um 10,6 Prozentpunkte gewesen, wodurch sich der Umlagesatz in kürzester Zeit mehr als verdreifacht hätte. Bei vielen kleineren kommunalen Zusatzversorgungskassen wäre ebenfalls mit steigenden Umlagesätzen $\mathrm{zu}$ rechnen gewesen. Eine solche Entwicklung hätte die Lohnnebenkosten im öffentlichen Dienst deutlich anwachsen lassen, was vor allem die Arbeitgeber unter Druck gesetzt hätte, die in der Vergangenheit keine Personalreduktion vorgenommen haben und somit auch nicht zur Ausdünnung der Umlagebasis beitrugen. Aber auch die Arbeitnehmer hätten empfindliche Einbußen hinnehmen müssen, da sie (zumindest bei der VBL) einen Teil der Umlage aus ihrem versteuerten Einkommen tragen (Puskás 2001, S. 312).

Diese Aussichten veranlassten die Tarifparteien, über eine grundlegende Reform der Zusatzversorgung zu verhandeln. Die Anstrengungen mündeten im Altersvorsorgeplan 2001 vom 13. November 2001 und fanden ihre formelle Umsetzung in der Unterzeichnung der Altersvorsorgetarifverträge (ATV/ATV-K) am 1. März 2002. Die Tarifparteien beschlossen dabei die Abkehr vom Gesamtversorgungssystem und einigten sich auf die Etablierung eines beitragsorientierten Betriebsrentensystems auf Basis von Versorgungspunkten.

\section{1 \\ Das neue Versorgungs- punktemodell}

Das neue Versorgungspunktemodell der Zusatzversorgung ist systematisch völlig anders aufgebaut als das abgelöste Gesamtversorgungssystem. Die Leistungszusage besteht nun nicht mehr aus der Sicherung eines bestimmten Versorgungsniveaus, sondern sie erfolgt beitragsorientiert. Dabei wird den Arbeitnehmern des öffentlichen Dienstes eine Leistung zugesagt, die sich ergäbe, wenn $4 \%$ ihrer versorgungs- pflichtigen Entgelte in ein vollständig kapitalgedecktes System einbezahlt würden (Präambel des ATV). Es wird demnach eine fiktive Beitragsleistung von $4 \%$ der Entgelte unterstellt, weshalb man von einer beitragsorientierten Leistungszusage spricht. Bestandteil dieser Zusage ist dabei auch eine garantierte (Mindest-)Verzinsung dieser Beiträge mit 3,25 \% während der Anwartschaftsphase und 5,25\% während der Rentenbezugsphase. Damit rückt das Versorgungspunktemodell auch von der endgehaltsbezogenen Betrachtung ab und honoriert die gesamte Arbeitsleistung im öffentlichen Dienst. Neben der höheren Transparenz verspricht dies auch eine größere Leistungsgerechtigkeit als im Gesamtversorgungssystem (Beckmann/Hebler 2008, S. 80).

In der Praxis werden jedem Arbeitnehmer am Ende eines Jahres in Abhängigkeit von seinem Entgelt und seinem Alter Versorgungspunkte gutgeschrieben. Diese Versorgungspunkte werden über die gesamte Dienstzeit hinweg aufsummiert und bestimmen beim Eintritt des Versicherungsfalls die Höhe der Zusatzrente. Die jährlich erdienten Versorgungspunkte $\left(V P_{t}\right)$ ermitteln sich nach folgender Formel:

$$
\begin{aligned}
V P_{t}= & \frac{\text { zusatzversorgungspflichtiges Entgelt }}{t} \\
& \times \text { Altersfaktor }_{t}
\end{aligned}
$$

Neben dem versorgungspflichtigen Entgelt ist vor allem der Altersfaktor maßgeblich. In Abhängigkeit vom Alter des Versicherten enthält er sowohl die zugesicherte Verzinsung als auch alle entscheidenden biometrischen Risiken, wie durchschnittlicher Rentenbeginn, durchschnittliche Rentenbezugsdauer sowie die Risiken von Erwerbsminderungs- und Hinterbliebenenrenten. Die zugrunde gelegten versicherungsmathematischen Risiken entstammen den Heubeck-Richttafeln 1998 (Heubeck 1998). Da die Altersfaktoren u. a. die zugesicherte Verzinsung abbilden, sind sie in jüngeren Jahren wesentlich höher als kurz vor Rentenbeginn. Das in der Formel enthaltene Referenzentgelt ist einheitlich auf $1.000 €$ festgesetzt. Zur Bestimmung der monatlichen Betriebsrente bei Eintritt des Versicherungsfalles werden die über die Jahre angesammelten Versorgungspunkte aufsummiert und mit dem sogenannten Messbetrag $(4 €)$ multipliziert.

Die Dynamisierung der Zusatzrenten wurde ebenfalls von der Beamtenversor- 
gung und sonstigen Bezugssystemen entkoppelt und erfolgt im neuen System einheitlich jedes Jahr zum 1. Juli um $1 \%$. Dadurch wird jedoch die Lohn- und Preisentwicklung bei der Dynamisierung nicht berücksichtigt, sodass bei entsprechend hoher Inflation der reale Wert der Rentenzahlungen sinkt. Dies ist der Tatsache geschuldet, dass der Dynamisierung in (fiktiv) kapitalgedeckten Versorgungssystemen enge Grenzen gesetzt sind. In (fiktiv) kapitalgedeckten Systemen muss die (wie auch immer dynamisierte) Zahlungsreihe der Renten in einem Barwert münden, der dem Barwert der (fiktiven) Beiträge entspricht. Eine höhere Dynamisierung führte damit - unter sonst gleichen Bedingungen - zu niedrigeren Einstiegsrenten.

Eine weitere Neuerung des neuen Versorgungspunktemodells ist, dass ein vorzeitiges Ausscheiden aus dem öffentlichen Dienst nicht mehr zu so drastischen Einschnitten bei der Altersversorgung führt wie zu Zeiten des Gesamtversorgungssystems. Verlässt ein Arbeitnehmer vor Eintritt des Versicherungsfalls den öffentlichen Dienst, so wird er unter Beibehaltung seiner kompletten Anwartschaft als beitragsfrei Versicherter bei der Zusatzversorgungskasse weitergeführt. Diese Neuregelung wird vor allem der gestiegenen Fluktuation im öffentlichen Dienst gerecht (Deutscher Bundestag 2005, S. 264, 270).

Die Anwartschaften im neuen Betriebsrentenmodell ergeben sich nicht ausschließlich aufgrund zusatzversorgungspflichtiger Entgelte, sondern es werden auch soziale Komponenten berücksichtigt. In diesen Fällen werden den Versicherten Versorgungspunkte zugesprochen, denen keine konkrete Arbeitsleistung zugrunde liegt (Marburger 2006, S.192). Im Einzelnen handelt es sich um die Zurechnung von Versorgungspunkten bei Elternzeit und frühzeitiger Erwerbsminderung bzw. frühzeitigem Tod. Eine weitere Möglichkeit, bei der den Versicherten Versorgungspunkte gutgeschrieben werden können, denen keine konkrete Arbeitsleistung zugrunde liegt, ist die Verteilung von Bonuspunkten. Diese ergeben sich, wenn aus den (fiktiven) Beiträgen der Versicherten nach Abzug der Kosten für die sozialen Komponenten und die Verwaltung ein (fiktiver) Überschuss erzielt wird (Seiter 2002, S. 512).

\begin{tabular}{cccccc}
\hline \multicolumn{6}{c}{ Tabelle 1: Altersfaktoren des Versorgungspunktemodells } \\
\hline Alter & Altersfaktor & Alter & Altersfaktor & Alter & Altersfaktor \\
\hline 17 & 3,1 & 26 & 2,3 & $40-41$ & 1,5 \\
18 & 3,0 & $27-28$ & 2,2 & $42-43$ & 1,4 \\
19 & 2,9 & 29 & 2,1 & $44-46$ & 1,3 \\
20 & 2,8 & $30-31$ & 2,0 & $47-49$ & 1,2 \\
21 & 2,7 & $32-33$ & 1,9 & $50-52$ & 1,1 \\
22 & 2,6 & 34 & 1,8 & $53-56$ & 1,0 \\
23 & 2,5 & $35-36$ & 1,7 & $57-61$ & 0,9 \\
$24-25$ & 2,4 & $37-39$ & 1,6 & $62+$ & 0,8 \\
\hline & & & & WSI MITTEILUNGEN \\
Quelle: § 8 III ATV. & & & &
\end{tabular}

\section{Übertragung der Renten
und Anwartschaften}

Eine der größten Herausforderungen bei der Umstellung von der Gesamtversorgung auf das Versorgungspunktemodell lag in der Übertragung der laufenden Renten und Anwartschaften. Problematisch war dabei, das rechte Maß zwischen Pauschalisierung und Vertrauensschutz zu finden. Denn einerseits mussten Millionen Renten und Anwartschaften umgestellt werden, andererseits war das Vertrauen der Versicherten in ihre Altersversorgung zu schützen.

Die am Umstellungsstichtag, dem 31.12.2001, laufenden Renten wurden betragsmäßig festgeschrieben und als Besitzstandsrenten weitergezahlt. Ihre Dynamisierung geschieht nun nicht mehr im Gleichklang mit der Anpassung der Beamtenpensionen, sondern erfolgt wie bei den neuen Betriebsrenten jährlich zum 1. Juli um $1 \%$. Somit wurden auch die laufenden Renten von den exogenen Bezugssystemen, der Beamtenversorgung und der gesetzlichen Rente, entkoppelt und entwickeln sich nun kalkulierbarer. Ferner werden die früher statischen Versicherungsrenten nun regelmäßig dynamisiert (Hügelschäffer 2002, S. 239).

Wesentlich problematischer gestaltete sich die Umstellung der Anwartschaften (Puskás 2003, S. 177). Durch die Fixierung auf das Endgehalt waren mitlaufende Anwartschaften im System der Gesamtversorgung nicht vorgesehen. Für eine Übertragung in das neue System musste jedoch ein Weg gefunden werden, wie die bestehenden Anwartschaften betragsmäßig ermittelt werden konnten, um dann als "fiktive“ Anwartschaften übernommen zu werden. Je nach zugebilligtem Vertrauensschutz wurden die Anwartschaften nach verschiedenen Verfahren ermittelt. Dabei erfolgte eine Hochrechnung auf den Zeitpunkt des erwarteten Eintritts des Versorgungsfalls zur Bestimmung der Gesamtversorgung. Von dieser hochgerechneten Gesamtversorgung musste die ebenfalls prognostizierte gesetzliche Rente abgezogen werden, um eine fiktive Zusatzrente bei Renteneintritt zu ermitteln. In Abhängigkeit von ihrer Dienstzeit wurde den Versicherten dann anteilig die fiktive Zusatzrente als Anwartschaft zuerkannt. Die Anwartschaften wurden in Versorgungspunkte umgerechnet und in Form einer Startgutschrift den Versorgungspunktekonten der Versicherten angerechnet. Da es keine mitlaufenden Anwartschaften im Gesamtversorgungssystem gab, konnte es auch kein eindeutig richtiges Verfahren zu deren betragsmäßiger Bestimmung geben. Aus diesem Blickwinkel müssen auch die langwierigen Gerichtsverfahren um die Startgutschriften gesehen werden (Hügelschäffer 2008, S. 254ff.; Konrad 2008, S. 296 ff.). Prinzipiell wurden bei der Ermittlung der Anwartschaften drei Gruppen unterschieden:

\subsection{RENTENNAHE JAHRGÄNGE}

Auf der einen Seite gab es die sogenannten rentennahen Jahrgänge, die alle Pflichtversicherten erfassten, die zum Umstellungsstichtag das 55. Lebensjahr vollendet hatten. Ihnen billigten die Tarifparteien einen besonderen Vertrauensschutz zu, da sie relativ kurz vor der Verrentung standen und somit weniger Möglichkeiten hatten, sich an die neuen Gegebenheiten anzupassen. Deswegen wurden ihre Anwartschaften unter weitgehender Anwendung des alten Rechts ermittelt. Dazu wurde die Gesamtversorgung bestimmt, die sie mit ihrem aktuellen Gehalt bei einem Renteneintritt mit 63 Jahren hätten erzielen können. Davon wurde die gesetzliche Rente abgezogen, die 
nach einer aktuellen Rentenauskunft ebenfalls auf das 63. Lebensjahr hochgerechnet wurde. Die so errechnete Zusatzrente wurde in Versorgungspunkte umgerechnet und um die Anzahl an Versorgungspunkten gekürzt, die noch im neuen Versorgungspunktesystem bis zum 63. Lebensjahr zu erzielen sind. ${ }^{3}$ Die Kritik an diesem Verfahren zielt vor allem darauf ab, dass der Berechnung das aktuelle anstelle des hochgerechneten Endgehalts zugrunde liegt. Diese Abweichung vom System der Gesamtversorgung führt zu tendenziell niedrigeren Anwartschaften. Ebenfalls kritisiert wird, dass die bereits beschlossenen Kürzungen in der gesetzlichen Rente nicht berücksichtigt wurden. Diese „Ungenauigkeiten" bei der Berechnung der Anwartschaften der rentennahen Jahrgänge hatten zur Folge, dass die zugrunde gelegten Versorgungsrenten ca. $5 \%$ bis $20 \%$ zu niedrig angesetzt wurden (Furtmayr/Wagner 2007, S. 303).

\subsection{RENTENFERNE JAHRGÄNGE}

Alle Pflichtversicherten, die nicht zu den rentennahen Jahrgängen zählten, waren den rentenfernen Jahrgängen zuzurechnen. ${ }^{4}$ Auf diese Gruppe fiel der weitaus größte Teil der Anwartschaften, weswegen hier auch Verfahren zur Ermittlung der Anwartschaften angewandt wurden, die stärker pauschalisierten als bei den rentennahen Jahrgängen. Die Berechnung ihrer Anwartschaften folgte einem anderen Prinzip als bei denen der rentennahen Jahrgänge und richtete sich nach den Vorgaben des $\$ 18$ Betriebsrentengesetz (BetrAVG), welcher bisher die Anwartschaften regelte, die Versicherten zugestanden wurden, die vorzeitig aus dem öffentlichen Dienst ausschieden. Dieser Regelung zufolge ermittelten die Kassen zunächst die maximal zu erreichende Gesamtversorgung, indem sie den maximalen Versorgungssatz von 91,25 \% (netto) bzw. $75 \%$ (brutto) auf das aktuelle Gehalt anwendeten. Davon wurde im nächsten Schritt die gesetzliche Rente nach dem steuerlichen Näherungsverfahren ${ }^{5}$ abgezogen, woraus sich die sogenannte Vollleistung ergab. Für jedes Jahr, das der Versicherte bis dato an versorgungsfähiger Zeit vorweisen konnte, wurden ihm 2,25 \% der Vollleistung als Anwartschaft zuerkannt. ${ }^{6}$ Die Kritik an diesem Vorgehen zielt vor allem auf das steuerliche Näherungsverfahren zur Schätzung der gesetzlichen Rente, da dieses Verfahren tendenziell höhere gesetzliche Renten generiert als unter realistischeren Annahmen zu erwarten wären (Furtmayr/Wagner 2007, S. 304). Im Weiteren wird kritisiert, dass der Satz von 2,25\% zur ratierlichen Anrechnung der Vollleistung dazu führt, dass die maximale Gesamtversorgung erst nach 44,44 Jahren erreicht wird, anstatt wie bisher bereits nach 40 Jahren. Zumindest in letzterem Punkt erkannte der Bundesgerichtshof einen Verstoß gegen den allgemeinen Gleichheitsgrundsatz (BGH IV ZR 74/06.), sodass es nun an den Tarifparteien liegt, eine rechtmäßige Umsetzung zur Ermittlung der Startgutschriften zu finden (Konrad 2008, S. 299).

\subsection{BEITRAGSFREI VERSICHERTE}

Die dritte Gruppe von Versicherten, deren Anwartschaften in das neue System übertragen werden mussten, sind die beitragsfrei Versicherten. Die betragsmäßige Ermittlung der Anwartschaft erfolgte bei ihnen bereits im alten System, weswegen hier die Umstellung weniger problematisch ausfiel.

\section{6 \\ Entwicklung der Finanzierung am Beispiel der VBL}

Neben den systemimmanenten Schwierigkeiten des Gesamtversorgungssystems führte vor allem die Entwicklung auf der Finanzierungsseite zum Systemwechsel in der Zusatzversorgung. Am Beispiel der größten Zusatzversorgungskasse, der VBL, wird im Folgenden aufgezeigt, vor welchen finanziellen Herausforderungen die zusätzliche Altersversorgung im öffentlichen Dienst steht. Denn auch wenn der Systemwechsel dafür sorgen konnte, dass das Leistungsrecht der Zusatzversorgung transparenter und besser kalkulierbar sowie die Unsicherheit im Zusammenhang mit der Rechtsprechung beseitigt wurde, werden die demografische Entwicklung und die Auswirkungen der Personalpolitik der Vergangenheit weiter auf die Finanzierungsseite einwirken.

Da durch den Systemwechsel der Einfluss der gesetzlichen Rente, der Beamtenpensionen sowie des Steuer- und Sozialabgabenrechts auf die Höhe der Zusatzrenten beseitigt wurde, ist für die zukünftige Ent- wicklung der Ausgaben vor allem die Entwicklung der Lebenserwartung und der Versichertenstruktur relevant. Ebenfalls reduziert wurde der Einfluss der Lohnentwicklung, da die laufenden Renten unabhängig von ihr jährlich um $1 \%$ erhöht werden und sich die Rentenhöhe nicht mehr wie bisher aus dem Endgehalt bestimmt. Lohnerhöhungen, die einem Versicherten kurz vor Renteneintritt gewährt werden, wirken sich daher nur noch marginal auf die zukünftige Rentenhöhe aus. Zur Bestimmung der zukünftigen Ausgaben der VBL müssen demnach vor allem die Entwicklung der Anzahl der Renten sowie die durchschnittliche Rentenhöhe betrachtet werden.

Ein Blick auf die Entwicklung der Rentenzahl (Abbildung 2) zeigt, dass bis zum Jahr 2030 ein kräftiger Zuwachs bei den Rentenempfängern zu erwarten ist. Während im Jahr 2008 noch insgesamt 1,2 Mio. Personen eine Zusatzrente der VBL erhielten, wird sich diese Zahl bis 2030 deutlich auf 1,94 Mio. erhöhen. Die Entwicklung in den Abrechnungsverbänden Ost und West verläuft dabei sehr unterschiedlich. Da die Zusatzversorgung in den neuen Ländern erst 1997 eingeführt wurde, befindet sich der Versicherten- und Rentenbestand dort noch in der Anlaufphase. Die Anzahl der Renten wird sich im Abrechnungsverband Ost bis zum Jahr 2030 um rund $300 \%$ auf dann insgesamt 0,35 Mio. Rentner erhöhen. Im Abrechnungsverband West steigt die Zahl der Renten von rund 1 Mio. (2008) auf 1,58 Mio. (2030). Dieser Anstieg ist vor allem der expansiven Personalpolitik im öffentlichen Dienst seit den 1970er Jahren geschuldet.

Nach dem Jahr 2030 verringert sich in beiden Abrechnungsverbänden aufgrund der fortschreitenden Personalreduktion im öffentlichen Dienst seit Beginn der 1990er

3 Eine Beispielrechnung findet sich in Langenbrinck/Mühlstedt (2007, S. 95).

4 Die Anwartschaften der Pflichtversicherten der neuen Länder wurden wegen der besonderen Bedingungen komplett nach den Regelungen der rentenfernen Jahrgänge ermittelt, da diese für die Betroffenen im Regelfall zu günstigeren Ergebnissen führten.

5 Das hier angewandte steuerliche Näherungsverfahren errechnet die zukünftige gesetzliche Rente unter Zugrundelegung des aktuellen Gehalts und unter der Annahme von 45 Versicherungsjahren. Detaillierte Berechnungsvorschrift unter BMF (2001).

6 Eine Beispielrechnung findet sich in Beckmann/ Hebler (2008, S. 161) 
Jahre die Zahl der Renten wieder. Im Jahr 2050 werden bei der VBL insgesamt rund 1,55 Mio. Personen eine Zusatzrente beziehen.

Für die weitere Entwicklung der Ausgaben der VBL ist neben der Entwicklung der Rentenzahl auch die durchschnittliche Rentenhöhe maßgebend. Abbildung 3 gibt die Entwicklung der durchschnittlichen Rentenhöhen der VBL bei einem unterstellten Einkommenstrend ab 2010 von $0,00 \%$ wieder. Durch die Ausblendung der Lohnentwicklung werden die strukturellen Effekte besser sichtbar.

Im Abrechnungsverband West wird die durchschnittliche Rentenhöhe von $360 €$ im Jahr 2008 auf rund $300 €$ im Jahr 2030 sinken. In dem Absinken der durchschnittlichen Rentenhöhe spiegelt sich u. a. der Systemwechsel wider. Die durchschnittliche Rentenhöhe sinkt hierbei durch die niedrigeren Renten der Neuzugänge. Dabei kommt zuerst der leistungssenkende Effekt aus der Überleitung der Anwartschaften zum Tragen, später schlägt sich das niedrigere Leistungsniveau des Versorgungspunktemodells nieder (Puskás/Thiel 2004, S. 8). Ein weiterer Grund für die niedrigeren Durchschnittsrenten ist in der gestiegenen Fluktuation im öffentlichen Dienst zu suchen, da sich dadurch der Anteil der Renten aus beitragsfreier Versicherung erhöht, denen tendenziell kürzere Versicherungszeiten und somit niedrigere Zusatzrenten zugrunde liegen. Die steigende Fluktuation führt somit zu einer höheren Rentenzahl und niedrigeren Rentenhöhen bei den Neuzugängen. Der Anstieg der Durchschnittsrenten nach dem Jahr 2030 ist auf die festgeschriebene Dynamisierung der Renten um jährlich $1 \%$ zurückzuführen.

Im Abrechnungsverband Ost dominiert hingegen der Anlaufeffekt im Rentenbestand. Während 2008 noch durchschnittliche Renten in Höhe von rund $95 €$ gezahlt werden, steigen diese kontinuierlich bis zum Jahr 2050 auf dann rund $430 €$ an. Dies liegt daran, dass den aktuellen Renten noch sehr geringe Versicherungszeiten zugrunde liegen und dass das Normalniveau erst mit der Zeit in den Rentenbestand hineinwachsen muss.

Abbildung 4 stellt die Entwicklung der Gesamtausgaben für Anstaltsleistungen der VBL nach verschiedenen Szenarien der Einkommensentwicklung im öffentlichen Dienst dar. Die schwarze Linie beschreibt den Verlauf der Ausgaben bei einem unterstellten Einkommenstrend von 0,00 \%. In
Abb. 2: Entwicklung der Rentenzahl bei der VBL ${ }^{1}$

2008 bis 2050 - in Tsd. -
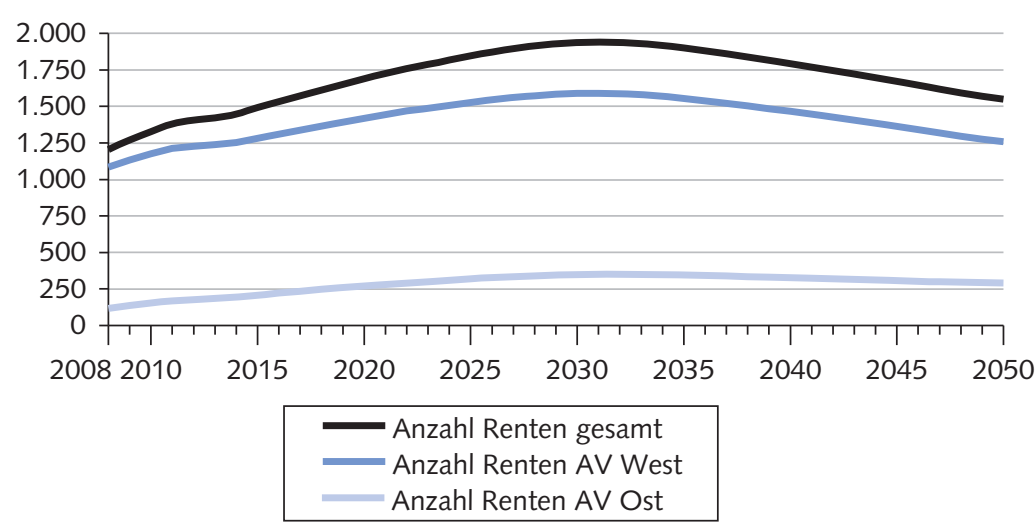

Versorgungskasse des Bundes und der Länder (Abrechnungsverband West).

Quelle: Hochrechnung der VBL 2008.

WSI MITTELUNGEN

\section{Abb. 3: Entwicklung der durchschnittlichen Höhe der \\ Zusatzrenten ${ }^{1}$ bei der VBL ${ }^{2} 2008$ bis 2050}

(Einkommenstrend ab 2010: 0,00 \%) - in Euro -

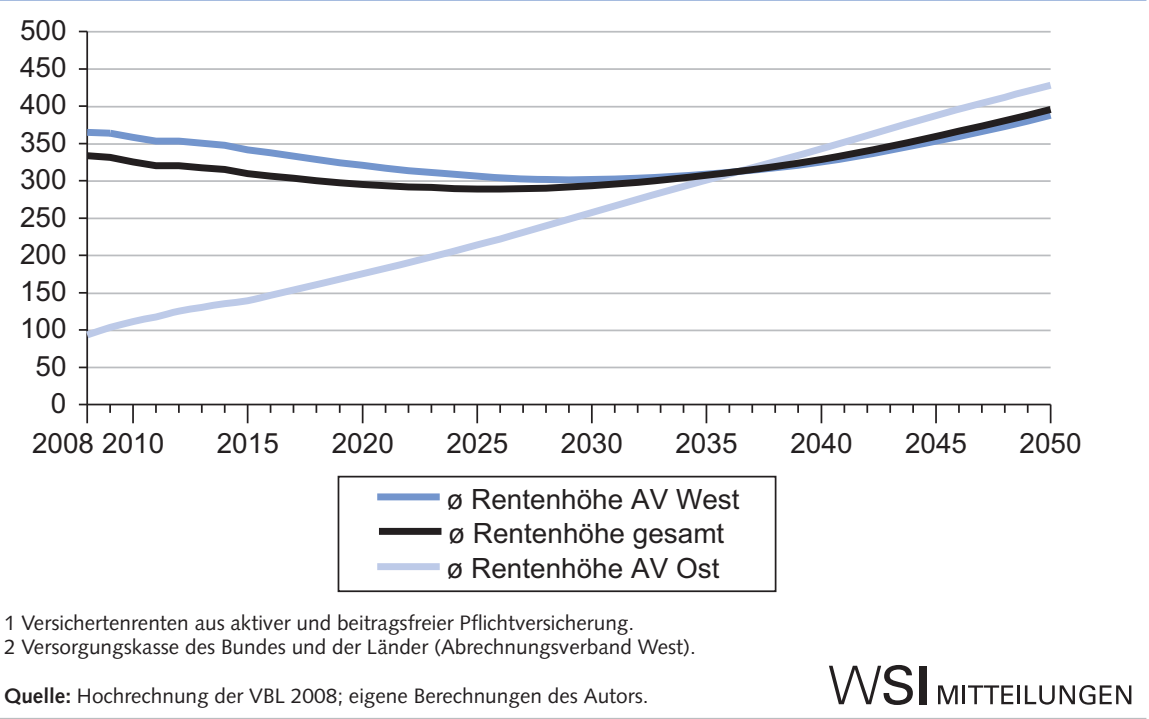

Quelle: Hochrechnung der VBL 2008; eigene Berechnungen des Autors.

WSI MITTELUNGEN

Abb. 4: Entwicklung der Anstaltsleistungen der VBL ${ }^{1}$ nach
verschiedenen Einkommenstrends 2008 bis 2050 - in Euro -

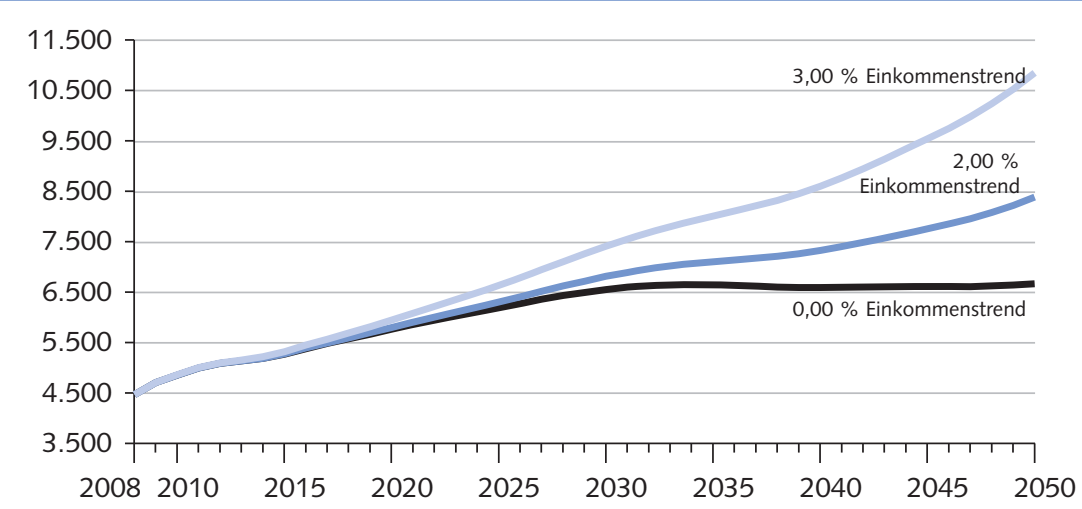

1 Versorgungskasse des Bundes und der Länder (Abrechnungsverband West). 
diesem Szenario steigen die Ausgaben von 4,5 Mrd. $€$ im Jahr 2008 bis auf 6,5 Mrd. $€$ im Jahr 2030 an. Dieses beträchtliche Ausgabenwachstum ist auf den starken Anstieg der Anzahl der Renten zurückzuführen. Im weiteren Verlauf bis zum Jahr 2050 bleiben die Ausgaben in etwa konstant. Dies liegt daran, dass sich in dieser Periode die sinkende Rentenzahl und die steigende durchschnittliche Rentenhöhe ungefähr ausgleichen. In den beiden anderen Szenarien ist dies nicht der Fall. Durch den unterstellten Einkommenstrend von 2,00\% (3,00\%) steigt die durchschnittliche Rentenhöhe mit fortschreitender Zeit stärker an, sodass gegen Ende des betrachteten Zeitraums im Jahr 2050 die Ausgaben bis auf 8,3 Mrd. € $(10,8$ Mrd. $€)$ ansteigen.

Abbildung 4 verdeutlicht, dass sich im neuen Versorgungspunktemodell die Lohnentwicklung erst verzögert in der Rentenhöhe niederschlägt. Bis zum Jahr 2025 sind die Unterschiede in den Ausgaben der verschiedenen Szenarien relativ gering, erst danach wachsen die Renten in den Bestand, deren Höhe deutlich durch die bessere Lohnentwicklung geprägt wurde.

\section{1 \\ Fazit und Ausblick}

Die Ablösung des Gesamtversorgungssystems war unter den gegebenen Bedingungen eine pragmatische Lösung für die anstehenden Finanzierungsschwierigkeiten der Zusatzversorgung. Mit der Etablierung des Versorgungspunktemodells konnten die Tarifparteien ein transparentes und besser kalkulierbares System erschaffen. Durch den Systemwechsel ist es gelungen, die finanziellen Risiken aus den systemimmanenten Abhängigkeiten zu beseiti- gen. Die Herausforderungen des demografischen Wandels und der Personalpolitik der Vergangenheit, die sich in der zukünftigen Versicherten- und Rentenstruktur niederschlagen, bleiben aber weiterhin bestehen. Eine teilweise Entspannung der Situation konnte jedoch durch die kostensenkende Übertragung der Anwartschaften und die Absenkung des Leistungsniveaus demnach zulasten der Arbeitnehmer erreicht werden. Der Effekt dieser Maßnahmen schlägt sich zukünftig in niedrigeren Zusatzrenten nieder und trägt dazu bei, den starken Anstieg der Rentenanzahl finanziell zu bewältigen. Die Frage, ob die Finanzierung, die gegenwärtig immer noch überwiegend im Umlageverfahren praktiziert wird, mit dem Systemwechsel nachhaltig gesichert werden konnte, muss an dieser Stelle offen bleiben und soll Gegenstand weiterer Forschung sein.

\section{LITERATUR}

Beckmann, J./Hebler, S. (2003): Zusatzversorgung nach dem Punktemodell für Arbeitnehmer des öffentlichen Dienstes, Stuttgart

Beckmann, J./Hebler, S. (2008): Zusatzversorgung für Arbeitnehmer des öffentlichen Dienstes, Stuttgart

Bundesministerium der Finanzen (BMF) (2001): Berücksichtigung von Renten aus der gesetzlichen Rentenversicherung ... (sog. Näherungsverfahren), Schreiben vom 5.10.2001, Berlin

Deutscher Bundestag (2005): Dritter Versorgungsbericht der Bundesregierung: Bundestagsdrucksache 15/5821

Ehrentraut, O./Raffelhüschen B. (2008): Demografischer Wandel und Betriebsrenten, in: Wirtschaftsdienst 8, S. 518-525

Fieberg, C. (2002): Neue Betriebsrente im öffentlichen Dienst, in: Betriebliche Altersversorgung (BetrAV) 3, S. 230-237

Furtmayr, H. L./Wagner C. (2007): Die Zusatzversorgung im öffentlichen Dienst - der heimliche Abschied von der Gesamtversorgung, in: Neue Zeitschrift für Sozialrecht (NZS) 6, S. 299-306

Heubeck, K. (1998): Richttafeln 1998, Köln

Heubeck, K./Rürup B. (2000): Finanzierung der Altersversorgung des öffentlichen Dienstes - Probleme und Optionen; Gutachten im Auftrag der Arbeitsgemeinschaft Kommunale und Kirchliche Altersversorgung (AKA) e. V., Sozialökonomische Schriften, Bd. 20, Frankfurt/M.

Hügelschäffer, H. (2002): Die neue Zusatzversorgung des öffentlichen Dienstes, in: Betriebliche Altersversorgung (BetrAV) 3, S. 237-241 Hügelschäffer, H. (2008): Die Entscheidung des BGH zur Systemumstellung in der Zusatzversorgung des öffentlichen und kirchlichen Dienstes eine Zwischenbilanz, in: Betriebliche Altersversorgung (BetrAV) 3, S. $254-$ 264

Kiefer, K.-H. (1996): Die Zukunft der Zusatzversorgung aus Arbeitgebersicht, in: Höfer, R. (Hrsg.): Die Zukunft der Altersversorgung des öffentlichen Dienstes, Stuttgart, S. 27-42
Konrad, M. (2008): Reform der Zusatzversorgung - Ende des Streits um die Startgutschriften in Sicht? Anmerkungen zum Urteil des Bundesgerichtshofs vom 14. November 2007 (IV ZR 74/06) zur Reform der Zusatzversorgung des öffentlichen Dienstes und zur Berechnung der Startgutschriften für die rentenfernen Jahrgänge, in: Zeitschrift für Tarifrecht (ZTR) 6, S. 296-303

Langenbrinck, B./Mühlstädt B. (2007): Betriebsrente der Beschäftigten des öffentlichen Dienstes : Einführung in die neue Zusatzversorgung, Heidelberg

Marburger, H. (2006): Die Versorgung der Beamten und anderweitig Beschäftigten im öffentlichen Dienst: Pension - Rente - Zusatzleistung, Berlin

Puskás, G. von (2001): Zukunftsperspektiven der Zusatzversorgung des öffentlichen und kirchlichen Dienstes, in: Betriebliche Altersversorgung (BetrAV) 4, S. 309-313

Puskás, G. von (2003): Die neue Zusatzversorgung erste Erfahrungen aus dem kommunalen und kirchlichen Bereich, in: Betriebliche Altersversorgung (BetrAV) 3, S. 177-181

Puskás, G. von/Thiel W. R. (2004): Die Rolle der Zusatzversorgung des öffentlichen Dienstes in der betrieblichen Altersversorgung, in: Die neue Zusatzversorgung im öffentlichen und kirchlichen Dienst, S. 7-8

Seiter, H. (2002): Reform der Zusatzversorgung im öffentlichen Dienst: Altersvorsorgeplan 2001, in: Betriebliche Altersversorgung (BetrAV) 6, S. 511-513

Seiter, H. (2003): Perspektiven der Zusatzversorgung, in: Betriebliche Altersversorgung (BetrAV) 3, S. 186-188

Stephan, A. (2002): Neuordnung der betrieblichen Altersversorgung im öffentlichen Dienst, in: Zeitschrift für Tarifrecht (ZTR) 2, S. 49-54 